\title{
Con historia hacia el futuro
}

\author{
With a history to the future
}

Fernando Estévez Abad

Inició su publicación en 1992, con la visión institucional de una sociedad que exigía conectarse con el mundo de la comunicación científica. Necesaria y siempre contemporánea con la medicina del momento, la Revista Ecuatoriana de Neurología está por cumplir tres décadas de actividad.

Llevada de la mano por reconocidos profesionales de la neurología ecuatoriana -el Dr. Óscar del Brutto y la Dra. Rocío Santibáñez- la REN, como órgano oficial de difusión de la Sociedad Ecuatoriana de Neurología, en los últimos años ha alcanzado su mejor condición en medio de las dificultades que implica comunicar de forma organizada, pero sobre todo rigurosa, los avances locales y -ahora- regionales del trabajo de los profesionales neurólogos y de aquellos que contribuyen al avance de las neurociencias y su búsqueda de respuestas.

El marco actual de reflexión bioética sobre lo que se publica científicamente pone de relieve algunos preceptos que deben cumplirse para garantizar el carácter certero de las comunicaciones de hallazgos científicos. Con sentido de aporte, pero sobre todo con el objetivo de que sean presentados a la comunidad científica y a la población en general en un marco que respete la verdad y oriente la búsqueda de mejoras en la calidad de vida de los pacientes (uno de los fines de la medicina). ${ }^{2}$ El inicio de cualquier medio de difusión científica implica grandes esfuerzos por quienes dan el primer paso, pero el trabajo tesonero se sustenta a lo largo del tiempo relacionando la dedicación de los profesionales y su necesidad de comunicar hallazgos con la seriedad del grupo humano de editores, revisores y diagramadores dedicados al mantenimiento de medios de difusión científica.

Los profesionales de la salud necesitamos comunicar nuestros hallazgos siguiendo estándares de calidad que, con el paso del tiempo, han ido transformándose en verdaderos hitos. Hoy, cualquier revista de calidad debe cumplir exigencias que le permitan: mantener impacto y prestigio, conseguir visibilidad y fácil acceso a su contenido, mantener puntualmente la periodicidad, sujetarse a sistemas rigurosos auditables y transparentes, además de ética y compromiso del equipo editorial, entre otros. ${ }^{3}$ Con estos esfuerzos cumplidos, se puede conseguir la indexación de la publicación en bases de datos reconocidas por la comunidad científica. Solamente manteniendo altos estándares de calidad puede sustentarse una permanencia que ofrezca al lector respaldo de calidad y ética en lo que se publica. Estos requerimientos ofrecen satisfacción a los autores; pero, sobre todo, contribuyen a mantener el sentido de responsabilidad en las publicaciones científicas como piedras angulares de la investigación. Son estos preceptos los que destacan y prevalecen en tiempos en los cuales hemos sido testigos de publicaciones falsas, incompletas o poco serias.

Este marco referencial de que es necesario comunicar y de que hay que hacerlo éticamente es el que ha construido la REN en casi treinta años de existencia. Las normas previstas incluso en documentos internacionales como la Declaración Universal sobre Bioética y Derechos Humanos (artículos 22, 23 y 24) rescatan la necesidad de que los avances científicos sean presentados a la comunidad, para que, además de que sean conocidos, puedan evaluarse y someterse a debate público.

En palabras del equipo del Observatorio de Bioética y Derecho de la Universidad de Barcelona (OBDUB), la comunicación científica debe ser por naturaleza una comunicación bidireccional. Se sugiere que, en las publicaciones científicas, se note el compromiso con la sociedad, de tal forma que sean las necesidades sociales
Presidente de la Sociedad Ecuatoriana de Neurología

Docente de la Universidad de Cuenca, Ecuador
Correspondencia:

Fernando Estévez Abad, MD PhD

E-mail: fer421@yahoo.com 
las que marquen buena parte de la agenda científica y de investigación. En el documento sobre diálogo entre ciencia y sociedad del OBD-UB ${ }^{4}$ se señala con claridad la intrínseca relación que debe mantener la comunidad científica con la sociedad. No se puede pensar que la comunicación científica es una mera transferencia pasiva de información. La comunicación seria y ética debe guiarse por la necesidad de aportar, de transformar, de facilitar la colaboración y de permitir la toma de decisiones, fines cuya trascendencia ha quedado desnudada globalmente por la pandemia de la COVID-19.

La REN ha alcanzado importantes logros en el contexto de las publicaciones científicas: el primero, sustentarse y sostenerse en el tiempo con seriedad y responsabilidad; el segundo, construir un sistema de comunicación serio, ético, con responsabilidad y compromiso de equipo; y el tercero, afrontar el siglo XXI como una de las pocas revistas ecuatorianas indexadas, conectadas con las necesidades de los autores $\mathrm{y}$, fundamentalmente, con los de la sociedad. Publica hallazgos relevantes de patologías neurológicas prevalentes, pero se orienta también hacia las necesidades de la sociedad, permitiendo así el debate inclusivo e interactivo entre ciencia y sociedad.

La Sociedad Médica Ecuatoriana de Neurología tiene, pues, una medalla por delante: un medio serio de difusión científica en el ámbito de las neurociencias con impacto y alcance regional. Y tiene, sobre todo, una muestra de la calidad de sus miembros y de su compromiso con la sociedad ecuatoriana y latinoamericana.

\section{Referencias}

1. Alarcón T. Evolución histórica de la Neurología Ecuatoriana. Revista Ecuatoriana de Neurología [Internet]. 1993 [cited 9 November 2021];2(1). Available from: http://revecuatneurol.com/wp-content/ uploads/2015/06/Evolucion.pdf

2. Los fines de la medicina (The Goals of Medicine) [Internet]. Fundacio Grifols. 2021 [cited 9 November 2021]. Available from: https://www.fundaciogrifols. org/es/web/fundacio/-/11-los-fines-de-la-medicinathe-goals-of-medicine-

3. Romero L. Criterios de calidad de las publicaciones. Revista Comunicar [Internet]. 2018 [cited 9 November 2021];. Available from: https://www.revistacomunicar.com/wp/escuela-de-autores/criterios-decalidad-de-las-publicaciones/

4. Casado M, Puigdomènech P. Document sobre els aspectes ètics del diàleg entre ciència i societat. [Place of publication not identified]: Publicacions i Edicions de la Universitat de Barcelona; 2018. 